\title{
Intensity Based Soundfield Reproduction over Multiple Sweet Spots Using an Irregular Loudspeaker Array
}

\author{
Huanyu Zuo, Prasanga N. Samarasinghe, Thushara D. Abhayapala \\ Audio \& Acoustic Signal Processing Group, The Australian National University, Canberra, Australia
}

\begin{abstract}
Intensity based soundfield reproduction methods are shown to provide impressive human perception of sound localization. However, most of the previous works in this domain either focus on a single sweet spot for the listener, or are constrained to a regular loudspeaker geometry, which is difficult to implement in real-world applications. This paper addresses both of the above challenges. We propose an intensity matching technique to optimally reproduce sound intensity at multiple sweet spots using an irregular loudspeaker array. The performance of the proposed method is evaluated by comparing it with the pressure and velocity matching method through numerical simulations and perceptual experiments. The results show that the proposed method has an improved performance.
\end{abstract}

Index Terms-Sound intensity, soundfield reproduction, multiple sweet spots, irregular loudspeaker array

\section{INTRODUCTION}

Spatial soundfield reproduction aims to create an immersive soundfield over a predefined spatial region so that the listener inside the region can experience a realistic but virtual replication of the original soundfield. To achieve this goal, classical techniques such as Ambisonics [1], Higher Order Ambisonics (HOA) [2]-[5] and Wave Field Synthesis (WFS) [6], [7] have been proposed. A multi-zone soundfield reproduction system was given in [8]. Recently, Shin et. al. proposed a velocity controlled soundfield reproduction technique to achieve an improvement of human sound localization performance by matching the particle velocity [9], [10]. All aforementioned works focus on matching either sound pressure or particle velocity, both of which are important quantities to describe a soundfield. In [11], authors introduced a soundfield reproduction method based on pressure and velocity matching to improve the listening experience at a non-central listening point. Another similar idea was pursued in [12], where the sound intensity, i.e., the product of the sound pressure and particle velocity, was reproduced to obtain the impression of the original sound at the origin. By extending [12], the localization performance was improved by means of HOA [13]. However, the works of [11], [12] and [13] are all restricted to a single reproduction position.

Array geometry is an important factor when we consider soundfield reproduction. Spherical arrays were extensively studied in literature [2], [14]. Gupta et. al. used a set of multiple circular arrays to reproduce 3D soundfield [15]. To

This work is supported by ARC Linkage Grant No. LP160100379 and the China Scholarship Council with the Australian National University. facilitate the implementation, a compact circular planar array of loudspeakers was proposed [16]. These spherical and circular geometries are all used for reproduction based on pressure matching. However, the performance of pressure matching degrades when the geometry of loudspeaker arrays becomes neither spherical nor circular. Particle velocity and intensity based reproduction methods can overcome this limitation, because the information of particle velocity can be controlled to improve soundfield reproduction [10]. A typical example with such a loudspeaker arrangement was reported [17], [18], where a rectangular loudspeaker array with same interelement spacing was used for the optimization of both sound pressure and particle velocity. An intensity based soundfield reproduction with a circular array of loudspeakers has shown good performance [19]. However, both the rectangular and the circular loudspeaker arrays are examples of evenly distributed array geometries.

In this work, we consider a practical reproduction system that is modeled to reproduce the desired soundfield at multiple sweet spots by driving an irregular loudspeaker array (e.g., in cinemas, we only need to create a good sound perception at seat positions rather than the whole region, and it is easier to implement if the loudspeaker array can be irregular). It is important to note that these sweet spots can be arbitrarily located in a predefined spatial region. An intensity matching technique is proposed to optimize the sound intensity at the sweet spots and the pressure and velocity matching is compared with the proposed technique in this paper.

\section{Problem Formulation}

Consider a set of distinct sweet spots, located at $\boldsymbol{x}_{b}=$ $\left(r_{b}, \theta_{b}, \phi_{b}\right)$ for the $b^{\text {th }}$ sweet spot, within a spherical region $\chi$ of radius $R$ as seen in Fig. 1. Note that the sweet spots can be arbitrarily chosen within $\chi$. Let there be an irregular array of loudspeakers with the $\ell^{\text {th }}$ loudspeaker located at $\boldsymbol{x}_{\ell}=\left(r_{\ell}, \theta_{\ell}, \phi_{\ell}\right)$ outside of $\chi$. The free field assumption is made and scattering is assumed to be negligible.

The complex acoustic intensity at any point $\boldsymbol{x}=(r, \theta, \phi)$ in $\chi$ can be represented by [20]

$$
\boldsymbol{I}(\boldsymbol{x}, k)=P^{*}(\boldsymbol{x}, k) \boldsymbol{V}(\boldsymbol{x}, k),
$$

where $\boldsymbol{I}(\boldsymbol{x}, k)=\left[I_{r}(\boldsymbol{x}, k), I_{\theta}(\boldsymbol{x}, k), I_{\phi}(\boldsymbol{x}, k)\right]$ and $\boldsymbol{V}(\boldsymbol{x}, k)=$ $\left[V_{r}(\boldsymbol{x}, k), V_{\theta}(\boldsymbol{x}, k), V_{\phi}(\boldsymbol{x}, k)\right]$ are sound intensity and particle velocity vector, respectively, $P(\boldsymbol{x}, k)$ is sound pressure, 
$k=2 \pi f / c$ is the wave number, $f$ is the frequency, $c$ is the speed of propagation, and $*$ stands for complex conjugate. The real part of $\boldsymbol{I}(\boldsymbol{x}, k)$ is often referred to as the active sound intensity, which represents the propagating sound energy and shows the direction of propagation at the point in space. The imaginary part of it, on the other hand, is referred to as the reactive sound intensity, which represents the non-propagating sound energy [21]. However, a soundfield in a spherical region is usually characterized by spherical harmonic coefficients of sound pressure (which can be extracted by using higher order microphones such as an EigenMike) instead of the spatial distribution of sound intensity. ${ }^{1}$

Given spherical harmonic coefficients of sound pressure for the desired soundfield $\left\{\alpha_{n m}(k)\right\}$ in the spherical region $\chi$, the number of arbitrary sweet spots $N_{P}$ from $\chi$, and an appropriate irregular loudspeaker array geometry, our objective is to calculate the spatial sound intensity (1) in $\chi$ and find the loudspeaker driving signals that reproduce the desired sound intensity at the sweet spots in the region.

\section{SPATIAL SOUND INTENSITY REPRESENTATION IN THE SPHERICAL HARMONIC DOMAIN}

We have recently formulated spatial sound intensity in the spherical harmonic domain [22], which decomposes (1) in terms of spherical harmonic functions. We have shown that the spatial distribution of sound intensity can be expressed directly from the spherical harmonic coefficients of sound pressure.

\section{A. Desired Sound Intensity}

Considering the spherical harmonic coefficients of sound pressure for the desired soundfield $\left\{\alpha_{n m}(k)\right\}$ are given, we can use the results of [22] to calculate the desired sound intensity at any point in $\chi$. According to the results in [22], [23], the components of desired sound intensity $\boldsymbol{I}^{d}(\boldsymbol{x}, k)=$ $\left[I_{r}^{d}(\boldsymbol{x}, k), I_{\theta}^{d}(\boldsymbol{x}, k), I_{\phi}^{d}(\boldsymbol{x}, k)\right]$ at any arbitrary $\boldsymbol{x}$ within $\chi$ can be decomposed, respectively, as

$$
\begin{aligned}
& I_{r}^{d}(\boldsymbol{x}, k)=\sum_{p=0}^{Q} \sum_{q=-p}^{p} S_{p q}^{(r, d)}(k, r) Y_{p q}(\theta, \phi), \\
& I_{\theta}^{d}(\boldsymbol{x}, k)=\sum_{p=0}^{Q} \sum_{q=-p}^{p} S_{p q}^{(\theta, d)}(k, r) Y_{p q}(\theta, \phi), \\
& I_{\phi}^{d}(\boldsymbol{x}, k)=\sum_{p=0}^{Q} \sum_{q=-p}^{p} S_{p q}^{(\phi, d)}(k, r) Y_{p q}(\theta, \phi),
\end{aligned}
$$

$$
\begin{aligned}
& S_{p q}^{(r, d)}(k, r)=\frac{i}{k \rho_{0} c} \sum_{n=0}^{N} \sum_{m=-n}^{n} \sum_{n^{\prime}=0}^{N} \sum_{m^{\prime}=-n^{\prime}}^{n^{\prime}}(-1)^{m+q} C_{n n^{\prime} p} \\
& \times \alpha_{n m}^{*}(k) \alpha_{n^{\prime} m^{\prime}}(k) j_{n}(k r) j_{n \prime}^{\prime}(k r) W_{1} W_{2},
\end{aligned}
$$

denoting spherical harmonic coefficients of desired sound intensity in $r$ direction, where $C_{n n^{\prime} p}=$ $\sqrt{(2 n+1)\left(2 n^{\prime}+1\right)(2 p+1) / 4 \pi}, \quad j_{n}(\cdot) \quad$ is the $n^{\text {th }}$ order

\footnotetext{
${ }^{1}$ The sound intensity can be estimated by measuring the particle velocity over the volume of interest point by point, which is time-consuming.
}

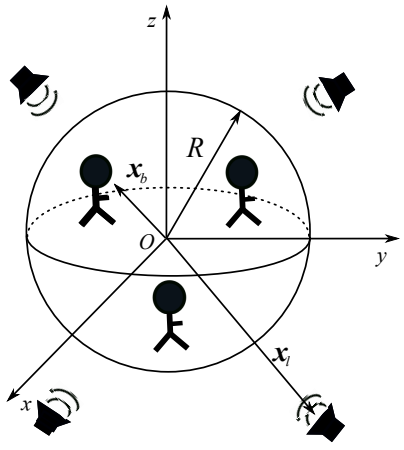

Fig. 1. Geometry of soundfield reproduction system in this paper. The sweet spots are arbitrarily chosen within the spherical region of radius $R$. An irregular array of loudspeakers is located outside the spherical region.

spherical Bessel function of the first kind, $j_{n}^{\prime}(\cdot)$ is the derivative of $j_{n}(\cdot)$ in terms of $r, \rho_{0}$ is medium density, $N=\lceil k e R / 2\rceil$ is the truncation limit of the soundfield orders [24],

$$
W_{1}=\left(\begin{array}{ccc}
n & n^{\prime} & p \\
0 & 0 & 0
\end{array}\right) \text {, and } W_{2}=\left(\begin{array}{ccc}
n & n^{\prime} & p \\
-m & m^{\prime} & -q
\end{array}\right)
$$

are Wigner 3-j symbols [25], $Q=2 N$ is truncation order for intensity expressions [22], and $Y_{p q}(\theta, \phi)=A_{p q} P_{p q}(\cos \theta) e^{i q \phi}$ is the spherical harmonic of order $p$ and degree $q$ with $A_{p q}=[(2 p+1)(p-q) ! /(4 \pi(p+q) !)]^{1 / 2}$, where $P_{p q}(\cos \theta)$ are the associated Legendre functions. In this work, we select the same truncation order for all components of sound intensity for simplicity, at the cost of relatively more error on the component in $\theta$ and $\phi$ direction compared to $r$ direction. Both $S_{p q}^{(\theta, d)}(k, r)$ and $S_{p q}^{(\phi, d)}(k, r)$ have similar expressions to (5) and are given in [22], [23].

\section{B. Reproduced Sound Intensity}

Assume that the loudspeakers in the reproduction system are point sources. The spherical harmonic coefficients of sound pressure due to the $\ell^{\text {th }}$ loudspeaker in free field can be written as [26]

$$
\beta_{n m}^{(\ell)}(k)=i k h_{n}\left(k r_{\ell}\right) Y_{n m}^{*}\left(\theta_{\ell}, \phi_{\ell}\right),
$$

where $h_{n}(\cdot)$ is the $n^{\text {th }}$ order spherical Hankel function of the first kind. By replacing $\alpha_{n m}(k)$ with $\beta_{n m}^{(\ell)}(k)$ in (2) and (5), we obtain sound intensity in $r$ direction due to the $\ell^{\text {th }}$ loudspeaker at $\boldsymbol{x}$ as

$$
I_{r}^{(\ell)}(\boldsymbol{x}, k)=\sum_{p=0}^{Q} \sum_{q=-p}^{p} S_{p q}^{(r, \ell)}(k, r) Y_{p q}(\theta, \phi),
$$

where $S_{p q}^{(r, \ell)}(k, r)$ are spherical harmonic coefficients of sound intensity in $r$ direction due to the $\ell^{\text {th }}$ loudspeaker.

In order to drive loudspeakers, we apply a weight to each loudspeaker. Therefore, the reproduced sound intensity in $r$ direction at $\boldsymbol{x}$ can be written as

$$
I_{r}^{a}(\boldsymbol{x}, k)=\sum_{\ell=0}^{N_{L}}\left|w_{\ell}(k)\right|^{2} I_{r}^{(\ell)}(\boldsymbol{x}, k),
$$

where $w_{\ell}(k)$ is the weight applying to the $\ell^{\text {th }}$ loudspeaker and $N_{L}$ is the number of loudspeakers. Here we assume incoherent superposition of loudspeaker signals, similar to max- $r_{E}$ decoding method in [12] and [13], which is used to reproduce the energy and acoustic intensity of the desired 
soundfield, and psychoacoustically to create the impression of the desired sound, especially for high frequencies (above 500 $\mathrm{Hz})$.

Similar to (8), the reproduced sound intensity in $\theta$ and $\phi$ directions at $\boldsymbol{x}$ can be expressed, respectively, as

$$
\begin{aligned}
I_{\theta}^{a}(\boldsymbol{x}, k) & =\sum_{\ell=0}^{N_{L}}\left|w_{\ell}(k)\right|^{2} I_{\theta}^{(\ell)}(\boldsymbol{x}, k), \\
I_{\phi}^{a}(\boldsymbol{x}, k) & =\sum_{\ell=0}^{N_{L}}\left|w_{\ell}(k)\right|^{2} I_{\phi}^{(\ell)}(\boldsymbol{x}, k) .
\end{aligned}
$$

Therefore, the reproduced intensity vector is $\boldsymbol{I}^{a}(\boldsymbol{x}, k)=$ $\left[I_{r}^{a}(\boldsymbol{x}, k), I_{\theta}^{a}(\boldsymbol{x}, k), I_{\phi}^{a}(\boldsymbol{x}, k)\right]$.

\section{INTENSITY BASED SOUNDFIELD REPRODUCTION OVER MULTIPLE SWEET SPOTS}

Given the desired soundfield, the reproduction problem is now reduced to calculate the loudspeaker driving signals/weights that can reconstruct the original sound intensity at all sweet spots. Pressure based least squares method [27] is a common approach to calculate loudspeaker weights in a soundfield reproduction system. However, for irregular loudspeaker arrays, this method may lead to errors, which is detrimental to the perception of the location of the source. In order to overcome this limitation, an alternative version of the least squares, the intensity matching technique, is given, which is based on the quantity of sound intensity closely linked with human perception of sound localization [28].

We equate the desired sound intensity to the reproduced sound intensity at the sweet spots to design the loudspeaker weights, i.e.,

$$
\boldsymbol{I}^{d}\left(\boldsymbol{x}_{b}, k\right)=\boldsymbol{I}^{a}\left(\boldsymbol{x}_{b}, k\right), \quad b=1,2, \ldots, N_{P},
$$

where $\quad \boldsymbol{I}^{d}\left(\boldsymbol{x}_{b}, k\right)=\left[I_{r}^{d}\left(\boldsymbol{x}_{b}, k\right), I_{\theta}^{d}\left(\boldsymbol{x}_{b}, k\right), I_{\phi}^{d}\left(\boldsymbol{x}_{b}, k\right)\right] \quad$ and $\boldsymbol{I}^{a}\left(\boldsymbol{x}_{b}, k\right)=\left[I_{r}^{a}\left(\boldsymbol{x}_{b}, k\right), I_{\theta}^{a}\left(\boldsymbol{x}_{b}, k\right), I_{\phi}^{a}\left(\boldsymbol{x}_{b}, k\right)\right]$. This can be expressed in matrix form as

$$
\boldsymbol{I}_{\boldsymbol{D}}=\boldsymbol{I}_{\boldsymbol{A}} \boldsymbol{W},
$$

where $\boldsymbol{I}_{\boldsymbol{D}}=\left[\boldsymbol{I}_{\boldsymbol{r}}^{\boldsymbol{d}}(k)^{T}, \boldsymbol{I}_{\boldsymbol{\theta}}^{\boldsymbol{d}}(k)^{T}, \boldsymbol{I}_{\boldsymbol{\phi}}^{\boldsymbol{d}}(k)^{T}\right]^{T}$ is a $6 N_{P}$ long vector with

$$
\begin{aligned}
& \boldsymbol{I}_{\boldsymbol{O}}^{\boldsymbol{d}}(k)=\left[\mathcal{R}\left\{I_{O}^{d}\left(\boldsymbol{x}_{1}, k\right)\right\}, \mathcal{I}\left\{I_{O}^{d}\left(\boldsymbol{x}_{1}, k\right)\right\}, \ldots, \mathcal{R}\left\{I_{O}^{d}\left(\boldsymbol{x}_{N_{P}}, k\right)\right\},\right. \\
& \left.\mathcal{I}\left\{I_{O}^{d}\left(\boldsymbol{x}_{N_{P}}, k\right)\right\}\right]^{T} ; \quad O \in\{r, \theta, \phi\},
\end{aligned}
$$

where $\mathcal{R}\{\cdot\}$ denotes the real part, $\mathcal{I}\{\cdot\}$ denotes the imaginary part, $\boldsymbol{W}=\left[\left|w_{1}(k)\right|^{2},\left|w_{2}(k)\right|^{2}, \ldots,\left|w_{N_{L}}(k)\right|^{2}\right]^{T}$ is a $N_{L}$ long vector and $\boldsymbol{I}_{\boldsymbol{A}}=\left[\boldsymbol{I}_{\boldsymbol{r}}^{\boldsymbol{a}},(k)^{T}, \boldsymbol{I}_{\boldsymbol{\theta}}^{\boldsymbol{a}}(k)^{T}, \boldsymbol{I}_{\boldsymbol{\phi}}^{\boldsymbol{a}}(k)^{T}\right]^{T}$ is a $6 N_{P}$ by $N_{L}$ matrix with

$$
\boldsymbol{I}_{\boldsymbol{O}}^{\boldsymbol{a}}(k)=\left[\begin{array}{ccc}
\mathcal{R}\left\{I_{O}^{(1)}\left(\boldsymbol{x}_{1}, k\right)\right\} & \cdots & \mathcal{R}\left\{I_{O}^{\left(N_{L}\right)}\left(\boldsymbol{x}_{1}, k\right)\right\} \\
\mathcal{I}\left\{I_{O}^{(1)}\left(\boldsymbol{x}_{1}, k\right)\right\} & \cdots & \mathcal{I}\left\{I_{O}^{\left(N_{L}\right)}\left(\boldsymbol{x}_{1}, k\right)\right\} \\
\vdots & \ddots & \vdots \\
\mathcal{R}\left\{I_{O}^{(1)}\left(\boldsymbol{x}_{N_{P}}, k\right)\right\} & \cdots & \mathcal{R}\left\{I_{O}^{\left(N_{L}\right)}\left(\boldsymbol{x}_{N_{P}}, k\right)\right\} \\
\mathcal{I}\left\{I_{O}^{(1)}\left(\boldsymbol{x}_{N_{P}}, k\right)\right\} & \cdots & \mathcal{I}\left\{I_{O}^{\left(N_{L}\right)}\left(\boldsymbol{x}_{N_{P}}, k\right)\right\}
\end{array}\right],
$$

TABLE I

LOUDSPEAKER LOCATIONS OF THE 8-CHANNEL ARRAY.

\begin{tabular}{cccc}
\hline Number & $r[\mathrm{~m}]$ & $\theta[\mathrm{deg}]$ & $\phi[\mathrm{deg}]$ \\
\hline 1 & 1 & 58.3 & 288 \\
2 & 1 & 58.3 & 216 \\
3 & 1 & 58.3 & 72 \\
4 & 1 & 90 & 18 \\
5 & 1 & 90 & 126 \\
6 & 1 & 121.7 & 324 \\
7 & 1 & 121.7 & 180 \\
8 & 1 & 148.3 & 72 \\
\hline
\end{tabular}

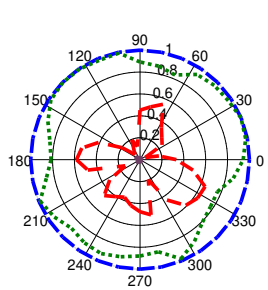

(a)

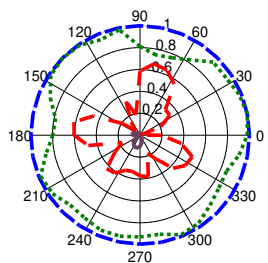

(c)

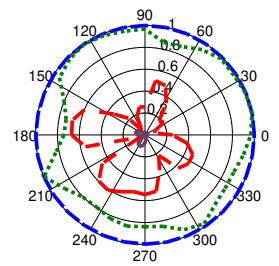

(e)

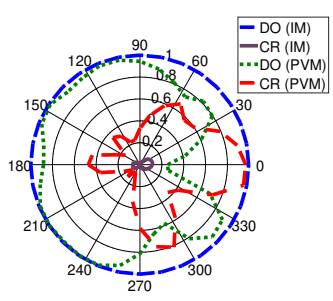

(b)

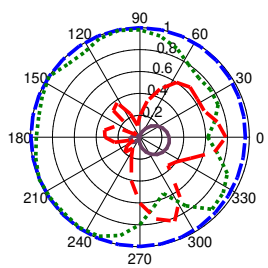

(d)

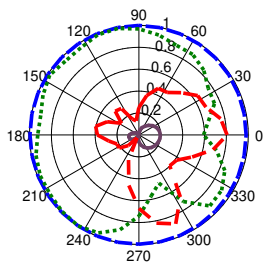

(f)
Fig. 2. Polar plots of DO and CR at sweet spots using the intensity matching (IM) and the pressure and velocity matching (PVM) for the virtual source moving on the horizontal plane ((a) sweet spot \#1, (c) \#2 and (e) \#3) and the vertical plane ((b) sweet spot \#1, (d) \#2 and (f) \#3).

where $O \in\{r, \theta, \phi\}$. Note that we deal with the real part and imaginary part of sound intensity separately as $\boldsymbol{W}$ is a real vector.

To minimize the difference between the desired sound intensity and the reproduced sound intensity, the problem is formulated as

$$
\min _{\boldsymbol{W}}\left\|\boldsymbol{I}_{\boldsymbol{A}} \boldsymbol{W}-\boldsymbol{I}_{\boldsymbol{D}}\right\|_{2}^{2}, \quad \text { s.t. } \boldsymbol{W} \geq 0,
$$

where $\|\cdot\|_{2}$ denotes the Euclidean norm, and $\boldsymbol{W} \geq 0$ means that each component of the vector $\boldsymbol{W}$ should be non-negative. A technique for solving this problem is known as non-negative least squares (NNLS) [29].

\section{EXPERIMENTAL EVALUATION}

\section{A. Experimental Setup and Criteria}

We consider a spherical region $\chi$ with radius $R=0.4 \mathrm{~m}$. The sweet spots can be selected at random within the region. 


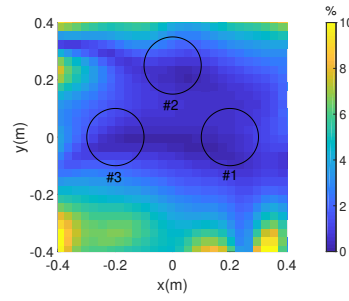

(a)

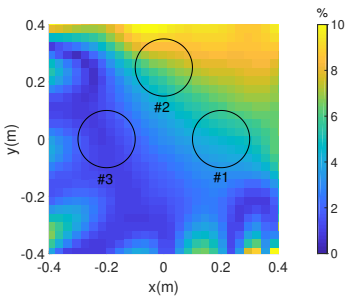

(b)
Fig. 3. The intensity direction error on plane $z=0$ controlled by (a) the intensity matching and (b) the pressure and velocity matching. Black circles denote human head zones around the sweet spots.

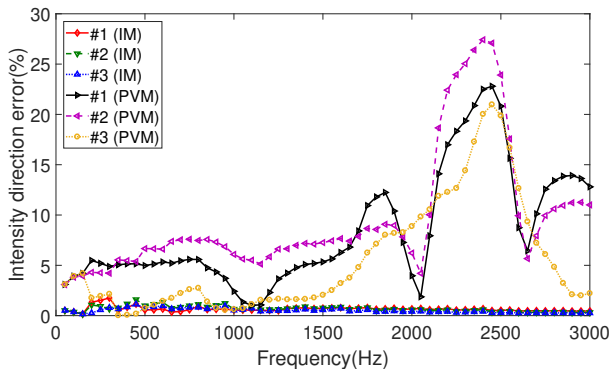

Fig. 4. The intensity direction error with respect to frequency.

In this case, we select sweet spot \#1, \#2 and \#3 located at $(0.2 \mathrm{~m}, \pi / 2,0),(0.25 \mathrm{~m}, \pi / 2, \pi / 2)$ and $(0.2 \mathrm{~m}, \pi / 2, \pi)$, respectively. Eight channels are selected to build the irregular loudspeaker array for reproduction in this work. The locations of the loudspeakers are given in Table I. The desired soundfield is produced by a virtual point source which is $2 \mathrm{~m}$ away from the origin. Sound speed $c$ is $343 \mathrm{~m} / \mathrm{s}$ and air density is 1.29 $\mathrm{kg} / \mathrm{m}^{3}$. To reproduce a soundfield with a realistic perception of the sound, it requires to ensure the reproduction of the direction of the sound. Therefore, we define two direction related quantities as follows

$$
\begin{aligned}
\mathrm{DO} & =\frac{\mathcal{R}\left\{\boldsymbol{I}^{a}(\boldsymbol{x}, k)\right\}}{\left\|\mathcal{R}\left\{\boldsymbol{I}^{a}(\boldsymbol{x}, k)\right\}\right\|_{2}} \cdot \frac{\mathcal{R}\left\{\boldsymbol{I}^{d}(\boldsymbol{x}, k)\right\}}{\left\|\mathcal{R}\left\{\boldsymbol{I}^{d}(\boldsymbol{x}, k)\right\}\right\|_{2}}, \\
\mathrm{CR} & =\frac{\mathcal{R}\left\{\boldsymbol{I}^{a}(\boldsymbol{x}, k)\right\}}{\left\|\mathcal{R}\left\{\boldsymbol{I}^{a}(\boldsymbol{x}, k)\right\}\right\|_{2}} \times \frac{\mathcal{R}\left\{\boldsymbol{I}^{d}(\boldsymbol{x}, k)\right\}}{\left\|\mathcal{R}\left\{\boldsymbol{I}^{d}(\boldsymbol{x}, k)\right\}\right\|_{2}},
\end{aligned}
$$

where $\cdot$ is dot product of two vectors and $\times$ is cross product of two vectors. DO and CR describe the difference between the reproduced direction and the desired direction. Ideally, if the direction of the sound at $\boldsymbol{x}$ is reconstructed perfectly, we have $\mathrm{DO}=1$ and $\mathrm{CR}=0$. Note that only the real part of complex acoustic intensity, which represents the propagation direction of sound energy, is considered here for evaluation. We also define the intensity direction error $\varepsilon$ to show the difference in angles between two vectors, which can be obtained from either of DO and CR. We represent it in terms of DO as

$$
\varepsilon=\cos ^{-1}(\mathrm{DO}) / \pi \times 100(\%) .
$$

The pressure and velocity matching is implemented by the least squares method.

\section{B. Experimental Results}

We first evaluate the reproduction methods for different positions of the virtual source. The source frequency is 600 Hz. Polar plots showing the results are given in Fig. 2. Among
TABLE II

AVERAGE ABSOLUTE PERCEIVED DIRECTION ERROR.

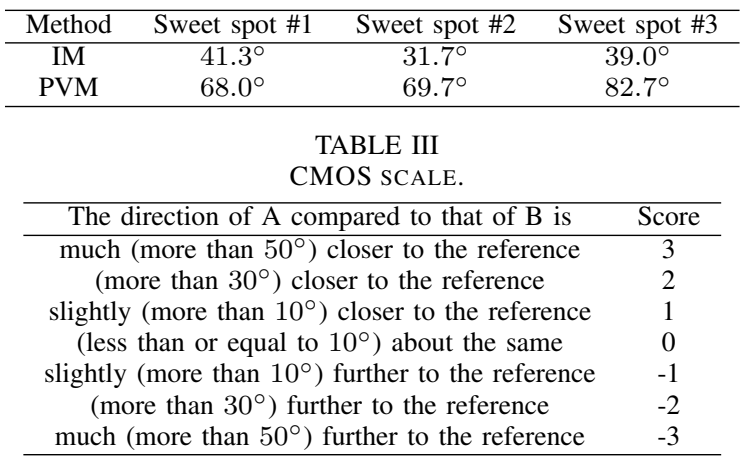

them, Fig. 2(a), Fig. 2(c) and Fig. 2(e) are the results for virtual source moving on the horizontal plane (the elevation is $\pi / 2$, and the azimuth changes from 0 to $2 \pi$ ). Figure 2(b), Fig. 2(d) and Fig. 2(f) are the results moving on the vertical plane (the azimuth is 0 and the elevation changes from 0 to $\pi$ for the upper half circle, and the azimuth is $\pi$ and the elevation changes from $\pi$ to 0 for the lower half circle). We observe that for all the evaluated positions of the virtual source, the intensity matching performs much better than the pressure and velocity matching at all sweet spots. We note that an irregular loudspeaker array cannot well cover all the incident directions, which leads to relatively poorer performance for the directions at which there are fewer loudspeakers.

The radius of human head is about $0.1 \mathrm{~m}$, therefore, we define a sphere with radius of $0.1 \mathrm{~m}$, centred by the sweet spot, as a human head zone. To evaluate the reproduction performance in human head zones, we calculate the intensity error on plane $z=0$ for both the intensity matching and the pressure and velocity matching, which is given in Fig.3. The black circles denote the human head zones on plane $z=0$. The virtual source is located at $(2 \mathrm{~m}, \pi / 2,2 \pi / 3)$. It shows that the intensity direction error controlled by the intensity matching is less than that controlled by the pressure and velocity matching in human head zones around the sweet spots. To examine the reproduction performance with respect to the change of frequencies, we also calculate the intensity direction error at the sweet spots for the frequency range from 50 to $3000 \mathrm{~Hz}$, which mostly covers the frequency range of human voice. The comparison between the intensity matching and the pressure and velocity matching is shown in Fig.4. The intensity direction error of intensity matching is no more than $3 \%$ within the frequency range at all the sweet spots. Compared with the intensity matching, the pressure and velocity matching has relatively larger error and the error noticeably fluctuates with frequency.

A perceptual listening test is carried out to validate the theory. The loudspeaker layout in Table I is built. The test samples consist of A, B and a reference, where the reference is the original sound signal produced by a loudspeaker located at ( $2 \mathrm{~m}, \pi / 2,2 \pi / 3)$, and A and B are reproduced by the eights channels using the intensity matching and the pressure and velocity matching, respectively. The hearing subjects do not have the knowledge of which method produces A, B or the 


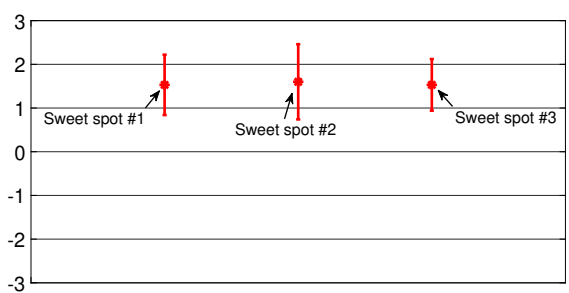

Fig. 5. CMOS scores for the three sweet spots with $95 \%$ confidence intervals.

reference. The hearing subjects are required to listen to $\mathrm{A}, \mathrm{B}$ and the reference at each sweet spot and record their perceived directions from 72 angles $\left((\theta, \phi)=\left(90^{\circ}, 5^{\circ} \times(n-1)\right)\right.$, where $n=1, \ldots, 72)$ on a provided answer sheet. During the test, the hearing subjects face positive $y$ direction and keep their heads at the center of each sweet spot. A clean speech of $5 \mathrm{~s}$ duration is used as a source signal and the sample rate adopted is $48 \mathrm{kHz}$ for signal inputs to all of the loudspeakers. The reference is used not only to compare with A and B, but also to identify an outlier within a data set (i.e., if the reference direction that a hearing subject perceives greatly deviates from its actual direction, the result from this subject is removed from the data set). A valid data set containing 15 hearing subjects is obtained after two outliers are removed. We calculate the average absolute perceived direction difference in angles between $\mathrm{A} / \mathrm{B}$ and the reference, which is given in Table II. It shows that the absolute error of the proposed method is less than that of the pressure and velocity matching for all the sweet spots. We also analyze the relative perceived direction error of A and B using Comparison Mean Opinion Score (CMOS), which has 7 levels shown in Table III. The higher the CMOS score is, the better the intensity matching is versus the pressure and velocity matching. The test results with $95 \%$ confidence intervals are given in Fig. 5. The average scores at the three sweet spots are $1.53,1.60$ and 1.53 , respectively, which means for this experimental setup the intensity matching can reproduce a better original sound image at all the sweet spots compared to the pressure and velocity matching.

\section{CONCLUSION}

In this paper, we have studied the intensity matching technique used for sound intensity reproduction at multiple sweet spots for irregular loudspeaker arrays to achieve a distributed listening area. We represent sound intensity in spherical harmonic coefficients of sound pressure and match it at all sweet spots using NNLS. The pressure and velocity matching has also been implemented for comparison. We demonstrate in the numerical simulations that the intensity matching is better than the pressure and velocity matching for overall reproduction performance. The perceptual experiment results also show that intensity matching can reproduce the direction of the sound at all sweet spots with less error than the pressure and velocity matching.

\section{REFERENCES}

[1] M. A. Gerzon, "Ambisonics in multichannel broadcasting and video," J. Audio Eng. Soc., vol. 33, no. 11, pp. 859-871, 1985.
[2] D. B. Ward and T. D. Abhayapala, "Reproduction of a plane-wave sound field using an array of loudspeakers," IEEE Trans. Speech Audio Process., vol. 9, no. 6, pp. 697-707, 2001.

[3] T. Betlehem and T. D. Abhayapala, "Theory and design of sound field reproduction in reverberant rooms," J. Acoust. Soc. Amer., vol. 117, no. 4, pp. 2100-2111, 2005.

[4] M. A. Poletti, "Three-dimensional surround sound systems based on spherical harmonics," J. Audio Eng. Soc., vol. 53, no. 11, pp. 10041025, 2005.

[5] Jens Ahrens and Sascha Spors, "Analytical driving functions for higher order ambisonics," in Proc. IEEE ICASSP, 2008, pp. 373-376.

[6] A. J. Berkhout, D. de Vries, and P. Vogel, "Acoustic control by wave field synthesis," J. Acoust. Soc. Amer., vol. 93, no. 5, pp. 2764-2778, 1993.

[7] A. J. Berkhout, "A holographic approach to acoustic control," J. Audio Eng. Soc., vol. 36, no. 12, pp. 977-995, 1988.

[8] Y. J. Wu and T. D. Abhayapala, "Spatial multizone soundfield reproduction," in Proc. IEEE ICASSP, 2009, pp. 93-96.

[9] M. Shin, F. M. Fazi, P. A. Nelson, and J. Seo, "Control of velocity for sound field reproduction," in Proc. 52th Int. AES Conf., 2013.

[10] M. Shin, P. A. Nelson, F. M. Fazi, and J. Seo, "Velocity controlled sound field reproduction by non-uniformly spaced loudspeakers," J. Sound Vib., vol. 370, pp. 444-464, 2016.

[11] S. Wang, R. Hu, S. Chen, X. Wang, B. Peng, Y. Yang, and W. Tu, "Sound physical property matching between non central listening point and central listening point for nhk 22.2 system reproduction," in Proc. IEEE ICASSP, 2017, pp. 436-440.

[12] D. Arteaga, "An ambisonics decoder for irregular 3-d loudspeaker arrays," in Proc. 134th AES Conv., 2013.

[13] D. Scaini and D. Arteaga, "Decoding of higher order ambisonics to irregular periphonic loudspeaker arrays," in Proc. 55th Int. AES Conf., 2014.

[14] Y. J. Wu and T. D. Abhayapala, "Theory and design of soundfield reproduction using continuous loudspeaker concept," IEEE Trans. Audio, Speech, Lang. Process., vol. 17, no. 1, pp. 107-116, 2009.

[15] A. Gupta and T. D. Abhayapala, "Three-dimensional sound field reproduction using multiple circular loudspeaker arrays," IEEE Trans. Audio, Speech, Lang. Process., vol. 19, no. 5, pp. 1149-1159, 2011.

[16] P. Chen, P. N. Samarasinghe, and T. D. Abhayapala, "3d exterior soundfield reproduction using a planar loudspeaker array," in Proc. IEEE ICASSP, 2018, pp. 471-475.

[17] M. Buerger, R. Maas, H. W. Löllmann, and W. Kellermann, "Multizone sound field synthesis based on the joint optimization of the sound pressure and particle velocity vector on closed contours," in Proc. IEEE WASPAA, 2015, pp. 1-5.

[18] M. Buerger, C. Hofmann, and W. Kellermann, "Broadband multizone sound rendering by jointly optimizing the sound pressure and particle velocity," J. Acoust. Soc. Amer, vol. 143, no. 3, pp. 1477-1490, 2018.

[19] H. Hacihabiboğlu and Z. Cvetković, "Panoramic recording and reproduction of multichannel audio using a circular microphone array," in Proc. IEEE WASPAA, 2009, pp. 117-120.

[20] J. A. Mann III, J. Tichy, and A. J. Romano, "Instantaneous and timeaveraged energy transfer in acoustic fields," J. Acoust. Soc. Amer, vol. 82, no. 1, pp. 17-30, 1987.

[21] H. Chen, T. D. Abhayapala, P. N. Samarasinghe, and W. Zhang, "Directto-reverberant energy ratio estimation using a first-order microphone," IEEE/ACM Trans. Audio, Speech, Lang. Process., vol. 25, no. 2, pp. 226-237, 2017.

[22] H. Zuo, P. N. Samarasinghe, T. D. Abhayapala, and G. Dickins, "Spatial sound intensity vectors in spherical harmonic domain," J. Acoust. Soc. Amer., vol. 145, no. 2, pp. EL149-EL155, 2019.

[23] H. Zuo, P. N. Samarasinghe, T. D. Abhayapala, and G. Dickins, "Erratum: Spatial sound intensity vectors in spherical harmonic domain [j. acoust. soc. am. 145 (2), el149-el155 (2019)]," J. Acoust. Soc. Amer., vol. 146, no. 1, pp. 164-165, 2019.

[24] H. Zuo, P. N. Samarasinghe, and T. D. Abhayapala, "Exterior-interior 3d sound field separation using a planar array of differential microphones," in Proc. IEEE IWAENC, 2018, pp. 216-220.

[25] F. W. J. Olver, "3j, 6j, 9j symbols," in NIST Handbook of Mathematical Functions. ch. 34. Cambridge University Press, 2010.

[26] P. Samarasinghe, T. Abhayapala, M. Poletti, and T. Betlehem, "An efficient parameterization of the room transfer function," IEEE/ACM Trans. Audio, Speech, Lang. Process., vol. 23, no. 12, pp. 2217-2227, 2015. 
[27] O. Kirkeby and P. A. Nelson, "Reproduction of plane wave sound fields," J. Acoust. Soc. Amer., vol. 94, no. 5, pp. 2992-3000, 1993.

[28] M. A. Gerzon, "Optimum reproduction matrices for multispeaker stereo," J. Audio Eng. Soc., vol. 40, no. 7/8, pp. 571-589, Jul. 1992.

[29] C. L. Lawson and R. J. Hanson, Solving least squares problems, vol. 15, Siam, 1995. 